\title{
Commentary
}

\section{Free perforator flaps on the peroneal vascular system}

\section{Gautam Biswas}

Department of Reconstructive Plastic Surgery, Tata Medical Center, New Town, Kolkata, West Bengal, India

Address for correspondence: Dr. Gautam Biswas, Department of Reconstructive Plastic Surgery, Tata Medical Center, New Town, Kolkata, West Bengal, India. E-mail: gautam.biswas@tmckolkata.co.in

he vascular anatomy of flaps has undergone a paradigm shift with the understanding of the anatomy of the perforators in cutaneous circulation. The territorial boundaries of perforators have been demonstrated by pioneers such as Manchot, Salmon and Taylor, amongst others. ${ }^{[-3]}$ Over 400 perforators have been defined with their potential of each supporting an unit of tissue.

The authors demonstrate one such flap to address a reconstructive option in composite oromandibular defects. Two patients are demonstrated in whom for composite defects of the head and neck a perforator based free flap has been combined with a fibular osteocutaneous flap from the same donor site i.e. lateral side of leg.

Conventional planning for composite oromandibular defects entails use of a single large flap, where the intervening segment can be de-epithelialised to mimic two skin "paddles." This protects the subdermal vascular plexus, and also preserves vessels communicating within the subcutaneous fat. Flap mobility is limited in this technique, being better suited for confluent defects as those near the commissure.

When the composite defect involves the mucosal and skin defects located at different areas, options are to use two separate free flaps or base each cutaneous component on

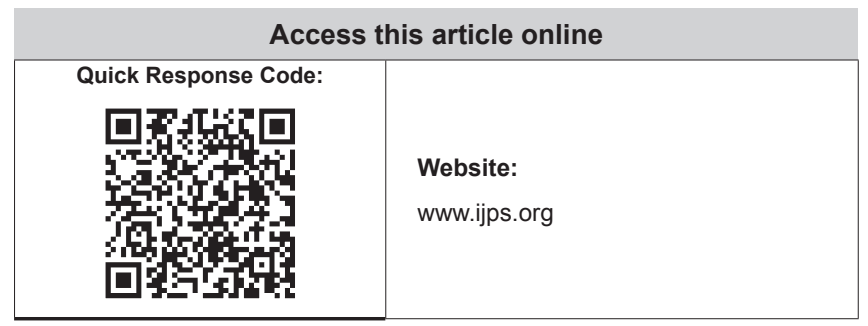

separate perforators (if available), completely islanding them, and hence allowing a greater mobility during inset [Figures 1 and 2].

The use of same donor source as clearly highlighted by the authors reduces donor site morbidity and operating time. Instrumentation, sutures and surgical skill now allow

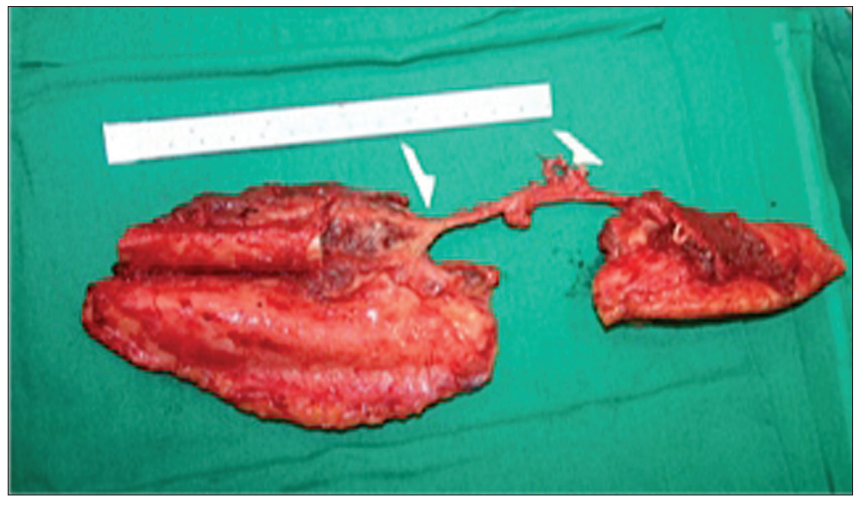

Figure 1: Double islanded fibula osteocutaneous flap, based on two separate perforators, arising from the peroneal trunk. Note the origin of the proximal perforator close to the origin of the peroneal vessel

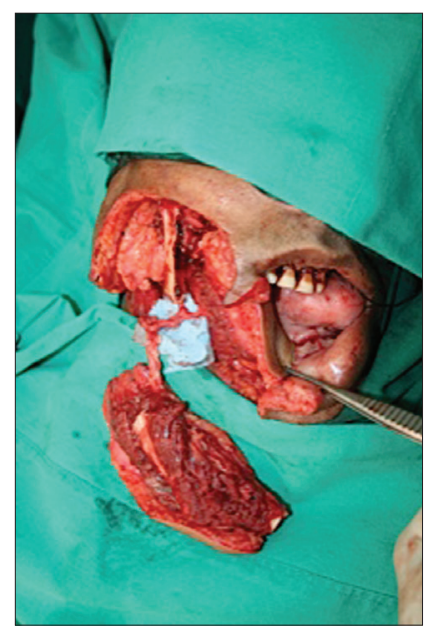

Figure 2: Inset of the flap into the defect demonstrating the flexibility in placing each component at the desired site 


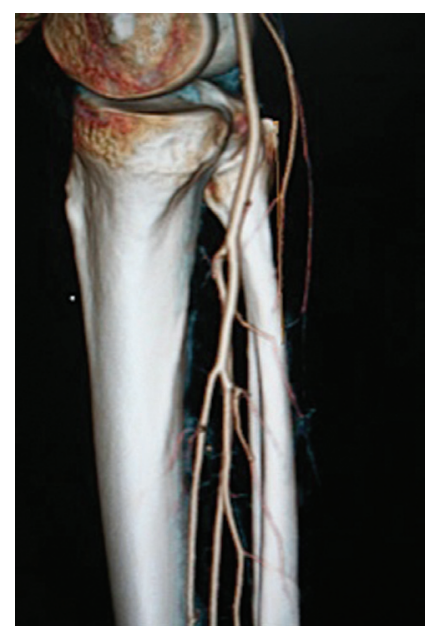

Figure 3: CT angiogram demonstrating the course and origin of the proxima peroneal perforator, arising from the common tibioperoneal trunk

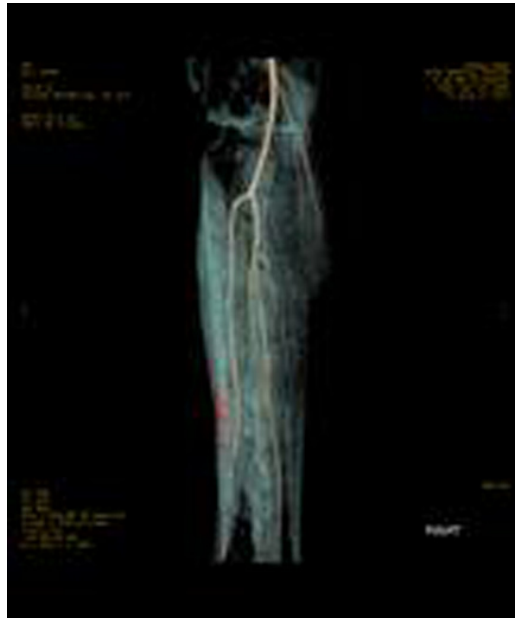

Figure 4: CT angiogram with subtraction of the bones to highlight the course and origin of the perforators

perforator anastomosis ( $<1 \mathrm{~mm}$ diameter), opening up the potential for such flaps. "Free-style free flaps," a term coined by Wei and Mardini, ${ }^{[4]}$ point to a flexible approach in planning after perforator identification. Though simplistic in concept, practically it is difficult to execute.

Consistency of such perforators, preoperative localisation, course and surface marking need to be studied, so that such procedures can be easily replicated.

We have been studying the perforators based on the peroneal vasculature using CT angiogram [Figures 3 and 4] and anatomical dissections.

A proximal perforator can be observed in a majority of the angiograms studied, situated $8-11 \mathrm{~cm}$ from the fibular head. The perforators have been found to take origin either from the peroneal trunk, the common tibioperoneal branch [Figures 3 and 4] of the popliteal or the popliteal vessels. The course of these perforators is long and in a majority has an intramuscular course, being accompanied by venae comitantes. The artery is of diameter between 0.6 and $0.8 \mathrm{~mm}$ with a pedicle length of around $7 \mathrm{~cm}$, allowing anastomosis wherever necessary.

Proximal perforators arise from the peroneal vessels soon after the tibioperoneal trunk bifurcates; this leaves a short pedicle length if retained with the peroneal vessels [Figure 1]. It can be divided and anastomosed as a second vascular input, allowing a greater flexibility.

The authors need to be commended for demonstration of their technique; however, further anatomical and clinical studies need to be detailed so that predictability of such flaps can be demonstrated.

Tedious intramuscular dissection of such perforators, combined with technical difficulties of anastomosis of small vessels will be a deterrent for frequent application of such flaps.

\section{REFERENCES}

1. Manchot $\mathrm{C}$. The cutaneous arteries of the human body. In: Ristic J, Morain WD, editors. New york: Springer-Verlag; 1983.

2. Salmon M. Arteres de la Peau. Paris (France): Masson; 1936.

3. Taylor GI, Palmer JH. The vascular territories (angiosomes) of the body: Eperimental study and clinical applications. Br J Plast Surg 1987;40:113-41.

4. Wei FC, Mardini S. Free style free flaps. Plast Reconstr Surg 2004;114:910-6.

How to cite this article: Biswas G. Free perforator flaps on the peroneal vascular system. Indian J Plast Surg 2012;45:466-7.

Source of Support: Nil, Conflict of Interest: Nil. 